\title{
Initial Proportion and Dynamic of B.1.1.7 SARS-CoV-2 in a Large City in the West of Germany
}

\author{
Simon D. Herkenrath ${ }^{a, b}$ Kyrill Boschung ${ }^{a} \quad$ Julia A. Nacov ${ }^{c} \quad$ Annette Heibges $^{c}$ \\ Britta Schroer ${ }^{c}$ Marcel Treml $^{b}$ Winfried J. Randerath ${ }^{a, b}$ \\ aBethanien Hospital gGmbH, Solingen, Germany; ${ }^{b}$ Institute for Pneumology at the University of Cologne, Solingen, \\ Germany; 'Stadtdienst Gesundheit, Solingen, Germany
}

\section{Keywords}

COVID-19 · SARS-CoV-2 · Alpha · Variant of concern · B.1.1.7

\begin{abstract}
Rationale: Several mutational variants of SARS-CoV-2 have been identified in the past months with increasing prevalence worldwide. Some variants, such as B.1.1.7, are of high relevance due to increased transmissibility, facilitating virus spread and calling for stricter containment measures. Objectives: The aim of this study was to examine proportion and dynamic of B-1.1.7 in SARS-CoV-2-positive samples in a large city in the west of Germany. Methods: Consecutive SARSCoV-2-positive samples from a local outpatient clinic, obtained over a period of 4 weeks (mid-January to mid-February 2021), were examined for the presence of the variant B.1.1.7. The size of B.1.1.7 infection clusters was compared with non-B.1.1.7 clusters. The transmissibility of SARS-CoV-2 variant B.1.1.7 was described based on corresponding cases of an infection cluster in a local child daycare centre. Results: Among 226 SARS-CoV-2-positive cases, B.1.1.7 was detected in 74 subjects (33\%). The 7-day moving mean of the B.1.1.7 proportion started at $20 \%$ and reached $50 \%$ only 3 weeks later. B.1.1.7 clusters comprised $10.7 \pm 12.1$ persons per clus-
\end{abstract}

Karger@karger.com www.karger.com/bmh

Karger $\stackrel{\text { ' }}{5}$

GOPEN ACCESS
(C) 2022 The Author(s)

Published by S. Karger AG, Basel

This is an Open Access article licensed under the Creative Commons Attribution-NonCommercial-4.0 International License (CC BY-NC) (http://www.karger.com/Services/OpenAccessLicense), applicable to the online version of the article only. Usage and distribution for commercial purposes requires written permission. ter, while non-B.1.1.7 clusters were considerably smaller (5.1 \pm 5.8). One specific B.1.1.7 infection cluster in a 40-children daycare centre started with one teacher leading to 11 infected children and 8 infections among teachers. The infection spread to 6 families and one other daycare centre, with a total 43 SARS-CoV-2-positive subjects. Conclusions: We found a rapid increase in the SARS-CoV-2 variant B.1.1.7 with larger infection clusters than non-B.1.1.7. These results suggested a rapid increase in the B.1.1.7 proportion and a renewed increase in the total number of SARS-CoV-2 infections for the time following the analysed period. Considering the rapid emergence and spread of viral variants, close monitoring of mutation events is essential. Therefore, routine whole-genome sequencing appears to be useful in addition to searching for known mutations.

(c) 2022 The Author(s) Published by S. Karger AG, Basel

\section{Background}

The SARS-CoV-2 pandemic has kept the world in suspense since early 2020 [1]. In the course of the pandemic, certain mutational variants of SARS-CoV-2 have been identified with increasing prevalence worldwide. In the 
first months of 2021, most notably variants B.1.351 and B.1.1.7 were on the rise. The former was first detected in South Africa in October 2020 and later designated variant "Beta", while the latter was first noticed in south England in September 2020 and designated "Alpha" when the World Health Organization suggested a nomenclature based on Greek letters to identify variants of concern and variants of interest [2]. Both variants are of high clinical relevance as they exhibit alterations of the receptor-binding domain in the spike glycoprotein which is believed to be associated with a higher transmissibility of the virus [3, 4]. Consequently, a steady and increasingly rapid increase in the proportion of these viral mutations has been recorded towards the end of 2020 [5, 6]. In England, the percentage of B.1.1.7. had already increased to over $95 \%$ by the beginning of February 2021 [2].

The proportion of SARS-CoV-2 mutational variants in Germany was not known until the end of 2020, and the Robert-Koch Institute reported a first case from 24 December [7]. Until then, systematic mutational analyses to investigate their incidence had not been performed. In view of the higher transmissibility and the development of infection numbers in England, particularly the proportion of B.1.1.7, a high number of unreported B.1.1.7 infections in Germany had to be assumed at the beginning of 2021.

We therefore aimed to:

- systematically examine SARS-CoV-2-positive samples regarding the proportion of mutation variant B.1.1.7 at a time when only few corresponding cases had been described,

- investigate the development of the proportion of variant B.1.1.7 over a period of 4 weeks,

- descriptively analyse local SARS-CoV-2 infection clusters regarding their size,

- and describe the transmissibility of SARS-CoV-2 variant B.1.1.7 based on corresponding cases of an infection cluster in a child daycare centre.

\section{Methods}

\section{Subject Population}

Consecutive SARS-CoV-2-positive samples, obtained in the outpatient clinic of Bethanien Hospital in Solingen over a period of 4 weeks (January 14 until February 11, 2021), were subjected to mutational analysis performed by Synlab laboratory in Leverkusen, Germany, to detect virus variant B.1.1.7. The local health authority of Solingen traced transmission routes and identified infection clusters. Clusters were defined as the emergence of $>2$ cases in the same time and space context. These data were anonymized prior to descriptive analysis, comparing subjects in whom infec- tion with variant B.1.1.7 was detected ("B.1.1.7”) against the group infected with non-B.1.1.7 virus variants ("non-B.1.1.7"). Infection-associated symptoms were documented at the time of virus sampling.

\section{Mutation Analysis}

The detection of variant B.1.1.7 was performed using a twostep process involving two of several different alterations in the spike protein, using the VirSNiP SARS-CoV2 Spike N501Y and del H69/V70 assays (TibMolBiol, Berlin, Germany) [8]. An initial melting curve analysis examined the presence of the N501Y mutation. This mutation has been described for the B.1.1.7 as well as the B1.351 variant. Based on the presence of N501Y, a second PCR, detecting the presence of the del H69/V70 mutation was performed. The del H69/V70 mutation is known to be present in the SARS-CoV-2 strands B1.1.7 as well as in the variant DK min V, first described in Denmark. If the N501Y mutation was detected, followed by a detection of the deletion H69/V70, variant B.1.1.7 was most likely present in the test material, and the samples were defined as such.

\section{Data Analysis}

Categorical data are presented as numbers and percentages while age is represented by median, first, and third quartiles. Age was tested for normal distribution using the Shapiro-Wilk test. Differences between groups were assessed using the Mann-Whitney $U$ test since normal distribution could not be assumed. Between-group differences regarding categorical data were assessed via a Fisher's exact test. All statistical analyses were performed in an explorative manner without specifying a threshold for statistical significance.

\section{Results}

Over a period of 4 weeks, 275 confirmed SARS-CoV-2 infections were documented. The mutation analysis of the corresponding samples was technically possible and led to definite results in 226 of these cases ( $82 \%$ ), forming the analysis cohort. Their median age was 37 years (range $2-90$ ), and 129 subjects (57\%) were female (Table 1). The B.1.1.7 variant was detected in 74 subjects $(33 \%)$, and the non-B.1.1.7 group consisted of 152 subjects (67\%). Both groups were of comparable age and gender distribution. The proportion of the B.1.1.7 variant increased over the course of 4 weeks. The 7 -day moving mean started at about $20 \%$, decreased during the next week, and then rapidly increased over another 7 days to about 50\% (Fig. 1).

Within the B.1.1.7 group, fourteen clusters were found. These clusters included an additional 76 subjects, who were identified as contact persons and tested positive for SARS-CoV-2, without their samples having been subjected to mutation analysis. This resulted in a total number of 150 SARS-CoV-2-positive subjects in all B.1.1.7 clusters, with individual cluster sizes ranging between 2 and 


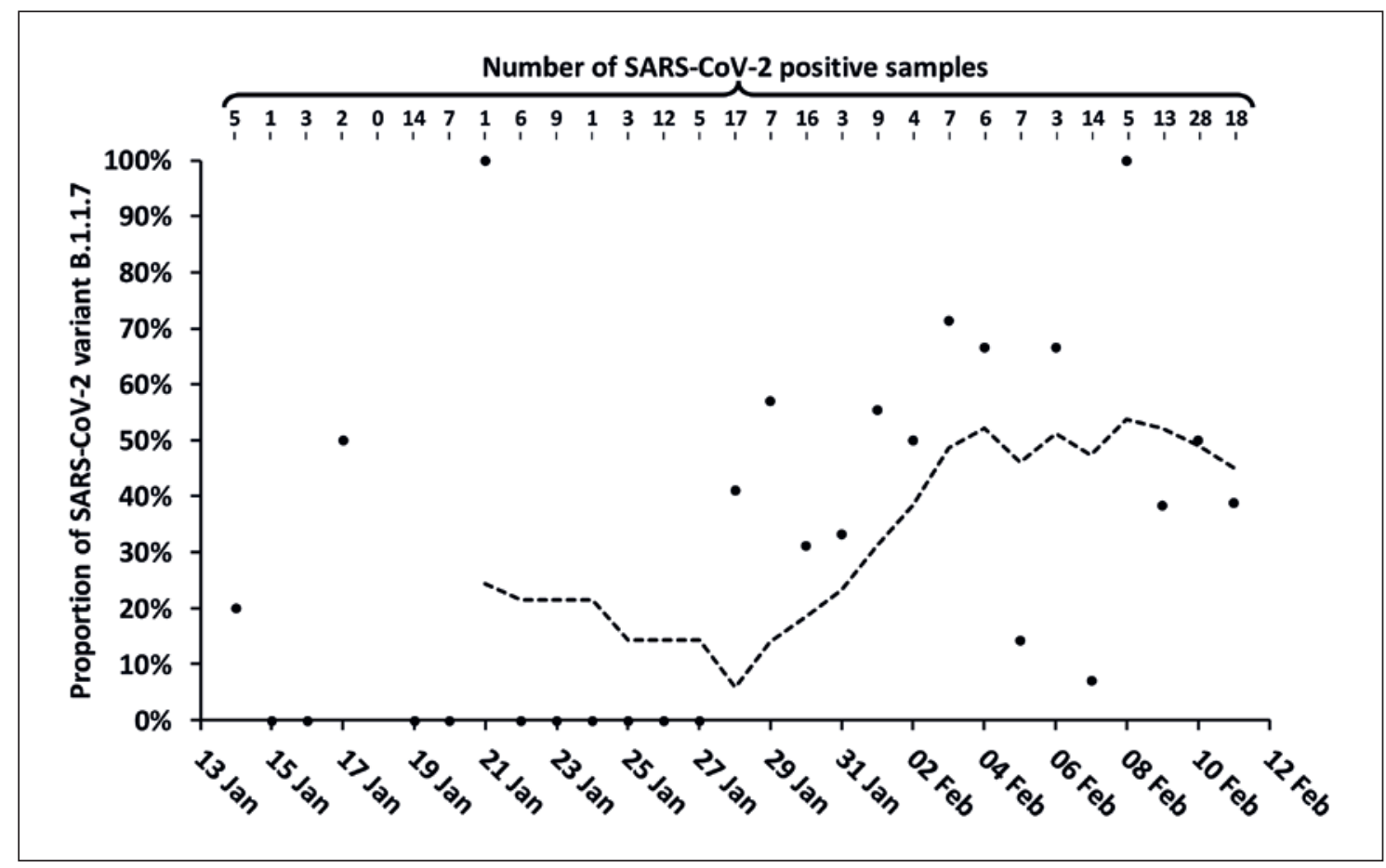

Fig. 1. Proportion of variant B.1.1.7 among SARS-CoV-2-positive samples during a period of 4 weeks. The data include all subjects with a definite result from the mutational analysis $(n=226)$. The dashed line represents the 7-day moving mean value.

Table 1. Anthropometric data and symptoms of the total group, subdivided into the B.1.1.7 and non-B.1.1.7 groups

\begin{tabular}{lllll}
\hline & Total & B.1.1.7 & Non-B.1.1.7 & $p$ value \\
\hline$n$ & 226 & $74(33)$ & $152(67)$ & - \\
Female & $129(57)$ & $45(61)$ & $84(55)$ & 0.427 \\
Age & $37[21 ; 52]$ & $38[23 ; 52]$ & $36[21 ; 52]$ & 0.770 \\
No symptoms & $85(38)$ & $33(45)$ & $52(34)$ & 0.864 \\
Cough & $74(33)$ & $22(30)$ & $52(34)$ & 0.464 \\
Pyrexia & $43(19)$ & $10(14)$ & $33(22)$ & 0.147 \\
Dyspnoea & $14(6)$ & $3(4)$ & $11(7)$ & 0.330 \\
Ageusia & $43(19)$ & $10(14)$ & $33(22)$ & 0.137 \\
Anosmia & $35(15)$ & $9(12)$ & $26(17)$ & 0.286 \\
Diarrhoea & $23(10)$ & $6(8)$ & $17(11)$ & 0.371 \\
Headache & $99(44)$ & $26(35)$ & $73(48)$ & 0.384 \\
Sore throat & $80(35)$ & $23(31)$ & $57(38)$ & 0.378 \\
Myalgia & $63(28)$ & $20(27)$ & $43(28)$ & 0.521 \\
Hyperhidrosis & $33(15)$ & $6(8)$ & $27(18)$ & 0.052 \\
Rhinorrhoea & $29(13)$ & $6(8)$ & $23(15)$ & 0.137 \\
Ague & $7(3)$ & $2(3)$ & $5(3)$ & 0.629 \\
Sudden onset & $44(19)$ & $10(14)$ & $34(22)$ & 0.087 \\
Strong feeling of illness & $17(8)$ & $4(5)$ & $13(9)$ & 0.303 \\
\hline
\end{tabular}

Shown are median [quartile 1; quartile 3] and number of patients (within-group percentages). 


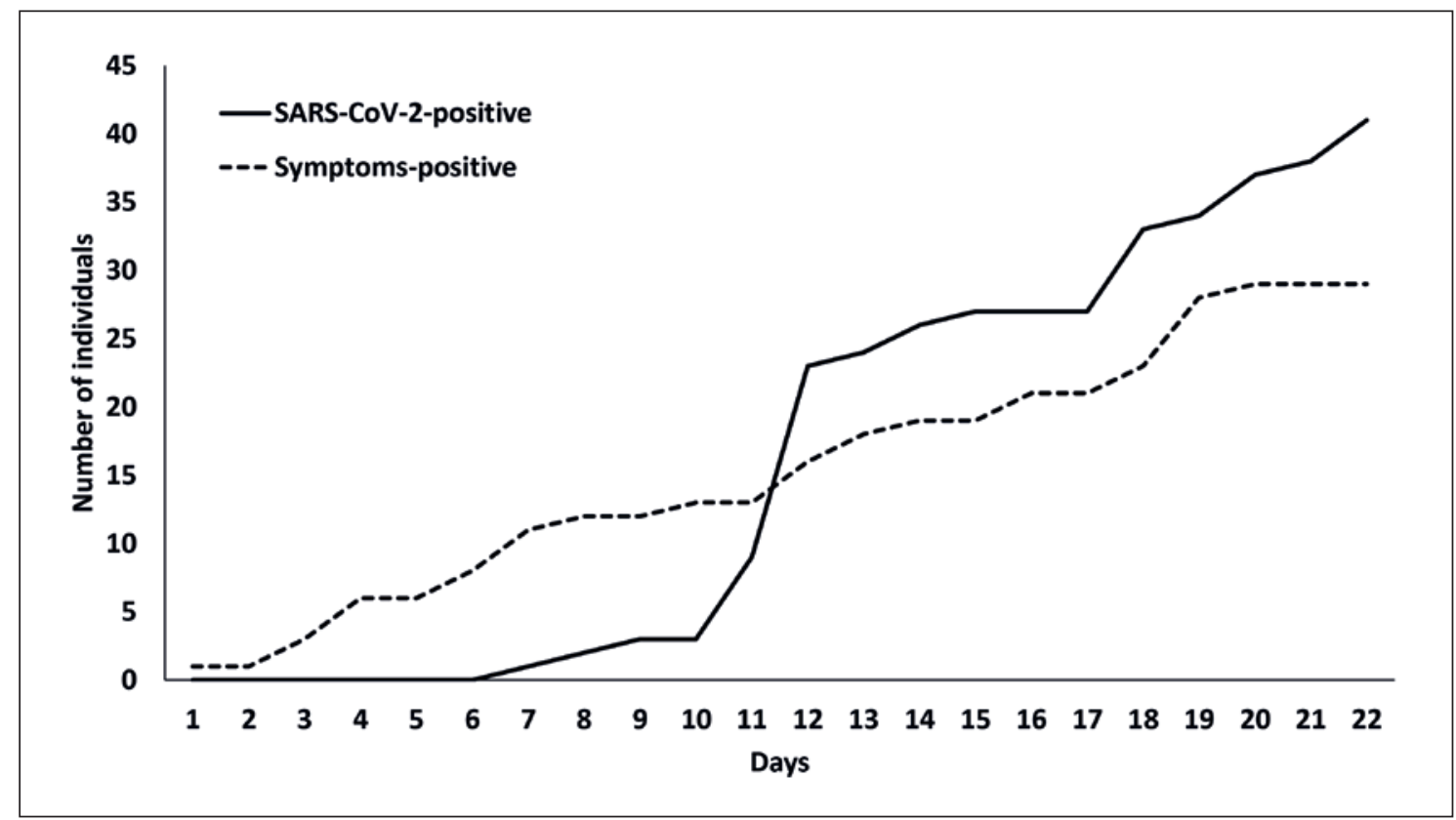

Fig. 2. Cumulative number of SARS-CoV-2-positive and symptom-positive individuals associated with a single infection event with the SARS-CoV-2 mutation B.1.1.7 which started in a daycare centre. The event starts with the onset of symptoms in the index person on Jan 29, 2021. The first 22 days are shown. The infection process was still developing after 22 days. Black line, cumulative number of people with a positive SARS-CoV-2 test; dashed line, cumulative number of people with symptoms.

40 subjects, resulting in a mean of 10.7 persons per cluster (standard deviation 12.1). Four of the fourteen clusters (29\%) consisted of $\geq 12$ subjects.

Subjects of the non-B.1.1.7 group, along with 177 additional contact persons also positive for SARS-CoV-2 but without mutation analysis (329 in total), were distributed in 65 clusters with a mean size of 5.1 subjects per cluster (standard deviation 5.8). As compared to the B.1.1.7 group, significantly fewer clusters consisted of $\geq 12$ subjects $(4 / 14,29 \%$ vs. $5 / 65,8 \%, p=0.047)$.

In the total group, $39 \%$ of the subjects were without symptoms. The three most common symptoms were headache $(44 \%)$, sore throat (35\%), and cough $(33 \%)$. Symptoms at the time of virus sampling did not significantly differ between B.1.1.7 and non-B.1.1.7 groups (Table 1).

\section{B.1.1.7 Transmissibility within a Child Daycare Centre-Associated Cluster}

The B.1.1.7 group included one cluster associated with a child daycare centre, which started with one member of their staff and resulted in 43 SARS-CoV-2-positive subjects in total (Fig. 2; online suppl. Fig. 1; for all online suppl. ma- terial, see www.karger.com/doi/10.1159/000519968). The daycare centre consisted of four groups (A-D) with 12, 15,12 , and 14 children, respectively. Furthermore, there were $7,3,4$, and 5 teachers or trainees in groups $\mathrm{A}, \mathrm{B}, \mathrm{C}$, and $\mathrm{D}$, respectively. The index person, a teacher from group A, showed first symptoms on 29 January and tested SARS-CoV-2-positive on 8 February, which led to a general order from the local health authority on the next day, requesting all daycare staff and children to be tested and quarantining all first-degree contact persons. Nevertheless, the infection continued to spread, with the index person infecting at least 11 other subjects directly, including the 5 family members of the index person's own household and 1 visitor at home. The latter transmitted the infection to his own mother. Furthermore, the head of the daycare, 2 teachers from group A, and 2 others from group $\mathrm{D}$ had become infected by the index person.

Either via the index person or secondarily via other teachers in the respective groups or the head of the daycare, a total of 11 children were infected $(4,2,4$, and 1 in groups $\mathrm{A}, \mathrm{B}, \mathrm{C}$, and $\mathrm{D}$, respectively), 3 of whom were symptomatic. In addition, four other teachers or trainees 
became infected so that there were $4 / 6,0 / 3,2 / 4$, and $2 / 5$ SARS-CoV-2-positive teachers in addition to the index person in the respective groups. Overall, the virus was transmitted to 6 families and to one other daycare centre, with a total of 15 more infected subjects, all of whom were adults. Within the child daycare centre with the index case, the raw attack rate was $28 \%$ (20 individuals with confirmed SARS-CoV-2 infection out of 72 at risk, excluding the index case). The attack rate in the second child daycare centre was $8 \%$ (3 SARS-CoV-2-positive individuals out of 38 at risk).

\section{Discussion}

Our analysis showed that the proportion of SARSCoV-2 variant B.1.1.7 increased rapidly over the course of 4 weeks starting mid-January and reaching approximately $50 \%$ by mid-February. This confirms the assumption of a high number of unreported cases during that period and a considerably dynamic development. The Robert-Koch Institute retrospectively reported a detection rate for variant B.1.1.7 of 5.6\% in the fourth calendar week of 2021 for Germany [9]. This rate increased to $22 \%$ in calendar week 6 (8-14 February) [10]. Based on this development and the experience from England at that time with variant B.1.1.7 accounting for more than $95 \%$ of all SARS-CoV2-positive cases, a similar progression could be expected for Germany [2]. Indeed, beyond the time period which constituted the data basis for our study, there was a further rapid increase in the B.1.1.7 proportion, which reached a value of around $90 \%$ at the beginning of April and remained stable on this level until the end of May [11]. The increasing proportion of the B.1.1.7 variant in the beginning of 2021 raised concerns as there was growing evidence for increased transmissibility, which is also supported by our observations, since the proportion of B.1.1.7 clusters with many infected individuals $(\geq 12)$ was significantly higher in our data compared with the non-B.1.1.7 group $[12,13]$. The numbers for Germany, provided by the Robert-Koch Institute, indeed show a marked increase in reported weekly cases of SARS-CoV-2-positive when the B.1.1.7 proportion surpassed the $20 \%$ mark. Furthermore, several studies from England recently published supported concerns as they found increased disease severity and mortality with the B.1.1.7 variant in addition to higher transmissibility $[12,14-16]$. These studies reached congruent results for the adjusted relative risk of death compared with non-B.1.1.7 infections, ranging from 1.59 to 1.67. Similar to B.1.1.7 with its higher transmissibility displacing other variants, by now, it has given way to variant B.1.617.2 (Delta) in many countries worldwide [17]. The increase in its proportion in Germany, starting around the end of April, shows a development similar to what was seen with B.1.1.7 and has reached a value of almost $75 \%$ in the first week of July 2021 [11]. In parallel, the proportion of B.1.1.7 inversely decreased over a period of 4 weeks, reaching $21 \%$ by the beginning of July. Challen et al. [14] assumed that the resulting number of deaths would scale linearly with the proportion of people infected with the new variant. However, as our data does not suggest the spectrum and severity of initial symptoms to differ between B.1.1.7 and non-B.1.1.7 infections, symptoms may not provide evidence of infection with one variant or the other. In fact, looking at the development in Germany, the increase in B.1.1.7 did not lead to a rise of the proportion of deaths, and instead, this number was constantly decreasing from mid-January onwards until July 2021 [11].

Our example of an outbreak in a child daycare centre clearly shows how the course of infections with the B.1.1.7 variant can develop, if the index person has ongoing social contacts after the onset of symptoms, even if only for a few days. The course of the pandemic in England has shown that the high number of SARS-CoV-2 infections, which reached their maximum at the end of 2020, could be significantly reduced by a stringent lockdown despite the increasing dominance of the B.1.1.7 variant. However, independent of current political decisions, it seems essential that in addition to the expansion and consistent use of testing, a self-reflective disease analysis is carried out to limit the spreading of SARS-CoV-2.

\section{Limitations}

The absolute incidence of SARS-CoV-2 as well as the relative incidence of variant B.1.1.7 could not be reliably determined based on our data since samples collected outside our test centre are not included in our analysis. This also constitutes a selection bias and impairs the generalization of our results. Furthermore, the identification of B.1.1.7 was based on the detection of N501Y and del H69/V70 mutations only as opposed to more accurate methods of variant identification, such as whole-genome sequencing. As we do not have complete information about potential infection spread within the families of all daycare children and teachers and assuming that symptomless children in particular and their families were not completely tested, we are unable to reasonably calculate the attack rate for all related households. Lastly, due to limited data availability, we were unable to compare attack rates of B.1.1.7 with non-B.1.1.7 variants.
Herkenrath/Boschung/Nacov/Heibges/ Schroer/Treml/Randerath 


\section{Conclusion}

Our analysis shows a rapid increase in SARS-CoV-2 variant B.1.1.7 with a higher proportion of large infection clusters than non-B.1.1.7 infections. This suggested a rapid increase in the proportion of B.1.1.7 and a renewed increase in the total number of SARS-CoV-2 infections for the time following the analysed period, which later could be confirmed by the actual development. Considering the high mutation frequency of the SARS-CoV-2 virus and the early and rapid spread of viral variants, close monitoring of mutation events is essential. For this purpose, routine whole-genome sequencing appears to be useful in addition to searching for known mutations.

\section{Statement of Ethics}

The study was conducted in accordance with all applicable laws and regulations, approved by the Ethics Commission of the University of Cologne (application no. 20-1252) and registered with ClinicalTrials.gov (NCT04521088). All subjects gave their written informed consent before their data was used in this analysis.

\section{Conflict of Interest Statement}

All authors declare no conflict of interest.

\section{Funding Sources}

This study did not receive any funding.

\section{Author Contributions}

Design of the work, acquisition and interpretation of the data, and drafting of the manuscript: S.D.H. and K.B.; Data analysis and revision of the manuscript: J.A.N., A.H., B.S., and M.T.; conception of the work, interpretation of the data, and revision of the manuscript: S.D.H. and W.J.R. All the authors are responsible for the final approval of the manuscript and accountable for all aspects of the work in ensuring that questions related to the accuracy or integrity of any part of the work are appropriately investigated and resolved.

\section{Data Availability Statement}

The data generated or analysed during this study will not be made publicly available. Enquiries concerning data access can be directed to the corresponding author.

\section{References}

1 Timeline: WHO's COVID-19 response [accessed 2021 Jul 12]. Available from: https:// www.who.int/emergencies/diseases/novelcoronavirus-2019/interactive-timeline.

2 Public Health England. https://assets.publishing.service.gov.uk/government/uploads/ system/uploads/attachment_data/ file/961299/Variants_of_Concern_VOC_ Technical_Briefing_6_England-1.pdf.

3 Leung K, Shum MH, Leung GM, Lam TT, Wu JT. Early transmissibility assessment of the N501Y mutant strains of SARS-CoV-2 in the United Kingdom, October to November 2020. Euro Surveill. 2021;26:2002106.

4 Pyke AT, Nair N, van den Hurk AF, Burtonclay P, Nguyen S, Barcelon J, et al. Replication kinetics of B.1.351 and B.1.1.7 SARS-CoV-2 variants of concern including assessment of a B.1.1.7 mutant carrying a defective ORF7a gene. Viruses. 2021;13:1087.

5 Vilar S, Isom DG. One year of SARS-CoV-2: how much has the virus changed? Biology. 2021;10(2):91.

6 Harvey WT, Carabelli AM, Jackson B, Gupta RK, Thomson EC, Harrison EM, et al. SARS$\mathrm{CoV}-2$ variants, spike mutations and immune escape. Nat Rev Microbiol. 2021;19:409-24.

7 Robert-Koch-Institut. Täglicher Lagebericht des RKI zur Coronavirus-Krankheit-2019 (COVID-19) Jul 28 26. https://www.rki.de/DE/Con-
tent/InfAZ/N/Neuartiges_Coronavirus/Situationsberichte/Dez_2020/2020-12-26-de.pdf.

8 Rambaut A, Loman N, Pybus O, Barclay W, Barrett J, Carabelli A, et al. Preliminary genomic characterisation of an emergent SARS$\mathrm{CoV}-2$ lineage in the UK defined by a novel set of spike mutations Jul $14 \mathrm{https} / / /$ virological.org/t/preliminary-genomic-characterisation-of-an-emergent-sars-cov-2-lineage-inthe-uk-defined-by-a-novel-set-of-spike-mutations $/ 563$.

9 Robert-Koch-Institut. Bericht zu Virusvarianten von SARS-CoV-2 in Deutschland, insbesondere zur Variant of Concern https:// www.rki.de/DE/Content/InfAZ/N/Neuartiges_Coronavirus / DES H / Bericht_ VOC_05022021.pdf.

10 Robert-Koch-Institut. 2. Bericht zu Virusvarianten von SARS-CoV-2 in Deutschland, insbesondere zur Variant of Concern https:// www.rki.de/DE/Content/InfAZ/N/Neuartiges_Coronavirus/DESH/Bericht_VOC_202102-17.pdf.

11 Coronavirus SARS-CoV-2: Tabelle zum VOC-Bericht - Anzahl und Anteile von VOC und VOI in Jul 14 https://www.rki.de/DE/ Content/InfAZ/N/Neuartiges_Coronavirus/ Daten/VOC_VOI_Tabelle.html.

12 Davies NG, Jarvis CI; CMMID COVID-19 Working Group; Edmunds WJ, Jewell NP,
Diaz-Ordaz K, et al. Increased mortality in community-tested cases of SARS-CoV-2 lineage B.1.1.7. Nature. 2021;593:270-4.

13 du Plessis L, McCrone JT, Zarebski AE, Hill V, Ruis C, Gutierrez B, et al. Establishment and lineage dynamics of the SARS-CoV-2 epidemic in the UK. Science. 2021;371:70812.

14 Challen R, Brooks-Pollock E, Read JM, Dyson L, Tsaneva-Atanasova K, Danon L. Risk of mortality in patients infected with SARSCoV-2 variant of concern 202012/1: matched cohort study. BMJ. 2021;372:n579.

15 Grint DJ, Wing K, Williamson E, McDonald HI, Bhaskaran K, Evans D, et al. Case fatality risk of the SARS-CoV-2 variant of concern B.1.1.7 in England, 16 November to 5 February. Euro Surveill. 2021;26:2100256.

16 Patone M, Thomas K, Hatch R, Tan PS, Liao $\mathrm{W}$, Coupland C, et al. Analysis of severe outcomes associated with the SARS-CoV-2 variant of concern 202012/01 in England using ICNARC Case Mix Programme and QResearch databases. medRxiv. 2021. Epub ahead of print.

17 World Health Organization. Weekly epidemiological update on COVID-19: Jul 142021 6. https: //www.who.int/publications/m/ item/weekly-epidemiological-update-on-covid-19---6-july-2021. 